\title{
Lapurdum
}

Euskal ikerketen aldizkaria | Revue d'études basques |

Revista de estudios vascos | Basque studies review

2| 1997

Numéro II

\section{Les relations internationales du nationalisme basque depuis son apparition jusqu'à la guerre civile espagnole (1890-1939)}

\section{Alexander Ugalde Zubiri}

\section{(2) OpenEdition}

\section{Édition électronique}

URL : http://journals.openedition.org/lapurdum/1838

DOI : 10.4000/lapurdum.1838

ISSN : 1965-0655

Éditeur

IKER

\section{Édition imprimée}

Date de publication : 1 octobre 1997

Pagination : 359-365

ISBN : 2-84127-142-0

ISSN : $1273-3830$

\section{Référence électronique}

Alexander Ugalde Zubiri, «Les relations internationales du nationalisme basque depuis son apparition jusqu'à la guerre civile espagnole (1890-1939) », Lapurdum [En ligne], 2 | 1997, mis en ligne le 01 septembre 2010, consulté le 29 janvier 2020. URL : http://journals.openedition.org/lapurdum/1838 ; DOI : 10.4000/lapurdum.1838 


\section{LES RELATIONS INTERNATIONALES DU NATIONALISME BASQUE DEPUIS SON APPARITION JUSQU'A LA GUERRE CIVILE ESPAGNOLE(1890-1939)*}

\section{L'action extérieure du nationalisme basque comme objet d'étude}

L'année 1995 marqua le centenaire du nationalisme basque, si l'on prend comme référence le 31 juillet 1895, date à laquelle s'est constitué le premier Bizkai-BurıBatzar ("Conseil Général de Bizkaia"), embryon de ce que sera le Parti Nationaliste Basque (PNB). Ce mouvement politique, apparu à la fin du XIX' siècle, a consolidé peu à peu sa doctrine, son programme et son organisation, gagnant en influence sociale et en présence électorale, de telle sorte qu'il s'est implanté comme une des composantes essentielles de l'histoire contemporaine basque et qu'il fait actuellement partie de la complexité de la réalité socio-politique basque à travers ses différentes formulations en matière d'idéologie, de politique et d'organisation.

Il est donc logique qu'un tel phénomène ait suscité l'attention des universitaires et des chercheurs dans différentes approches et disciplines, puisque c'est un des thèmes les plus abordés dans le domaine du renouvellement historiographique basque. On peut citer d'importantes recherches relatives au nationalisme basque (A. Elorza, J. Corcuera, J-C. Larronde, J-J. Solozabal, J-L. de la Granja, J. Apalategi, S. de Pablo, L. Mees, M. Ugalde, F. de Meer, I. Chueca, J. Azcona, A. Martínez Peñuela, P. Waldmann, . Heiberg...). Malgré cela, on peut noter comme une des carences existantes le peu de recherches ayant une approche intemationale. Dans ce domaine, je dois citer. sans prétendre être exhaustif, les apports de José Luis de la Granja (analyse du pacte Galeuzca - accord entre les nationalismes catalan, galicien et basque - et l'engagement des signataires d'un travail d'ensemble de « propagande et action internationale »), Xosé Estévez (relations entre les nationalismes périphériques péninsulaires, une de ses réflexions porte sur la conception « internationale » de ces alliances); Xosé M. Núñez Seixas (thèse doctorale sur les nationalités dans l'Europe de l'entredeux guerres et la « protodiplomatie » catalane, galicienne et basque lors du Congrès des Nationalités Européennes), Daniele Conversi (incidence des faits internationaux dans les nationalismes basque et catalan), José Maria Lorenzo Espinosa (influence du nationalisme irlandais dans le nationalisme basque), Juan Carlos Jiménez de Aberasturi (recueil documentaire des activités du Conseil National Basque à Londres), Koldo San Sebastián (recueil des actes de la Délégation basque à New York) et, à partir d'une perspective des Relations Internationales. Iñaki Aguirre Zabala (modèles historiques d' un espace basque intégré et son articulation avec les autres espaces internationaux). 
A partir de cet êtat de la question, j'ai rédigé une Thèse Doctorale qui contribue à analyser et évaluer l'action extérieure basque (objet de base de l'étude), adoptant un cadre historique suffisamment étendu sur une période de cinquante ans (1890-1939). Il s`agit de vérifier aussi bien les aspects théoriques que la matérialisation pratique d'une telle action et ainsi répondre à une interrogation centrale : est-ce que le nationalisme basque dispose d'un modèle défini d'action extérieure durant la période citée ?

\section{Vérification d'une triple hypothèse}

Je considère avoir réuni les données et les éléments suffisants pour démontrer que le nationalisme basque réussit à mettre en oeuvre un ensemble d'activités qui correspondent à une politique internationale, avec deux faces complémentaires : d'une part l'élaboration d'une ligne théorique d'action extérieure ; et d'autre part, son application sur le terrain avec l'établissement de relations internationales.

L'étude de ce processus a permis de vérifier la réalité de cette triple hypothèse conduite initialement, abordant méthodologiquement trois approches :

1) Du point de vue de l'Histoire : le nationalisme basque, comme mouvement politico-social, est structuré et organisé en plusieurs formations politiques et en différentes phases. Depuis sa naissance à la fin du XIX' siècle jusqu’à la fin des années trente, il déploya une action extérieure croissante. Lors du premier nationalisme, l'activité fut faible et irrégulière, ensuite elle se développa jusqu'à atteindre lors des années républicaines, un niveau que je qualifie d'important et de significatif.

2) Dans une perspective de pensée politique : la nature du nationalisme basque est de diriger son action vers une dimension internationale. Sa doctrine, ses principes inspirateurs, ses objectifs et ses programmes le conduisirent à situer son projet politique en référence au cadre européen et au contexte international. En premier lieu, il dut se stabiliser politiquement, socialement et électoralement dans le Pays Basque ; en second lieu, il dut déposer ses demandes devant le gouvernement central et participer à la dynamique politique de l'Etat espagnol ; par extension et en troisième lieu, il dut se situer sur la scène internationale, suivant en grande partie le sillage des mouvements nationaux. En définitive, j’enregistre une cohérence entre la doctrine du nationalisme basque et sa dynamique de projection extérieure.

3) Du point de vue des Relations Internationales : le nationalisme basque exerça une action extérieure où l'on peut relever les différents indicateurs qui composent un modèle précis dans son évolution historique. On peut prendre les indicateurs d'une manière séparée ou dans leur ensemble. En ce sens, il s'agit d'une action extérieure typique d'un mouvement politique, c'est-à-dire une diplomatie non gouvernementale. Le plus important de cette action extérieure fut de recevoir la reconnaissance internationale du nationalisme comme mouvement politique et d'aspirer, en une vision du futur, à la reconnaissance internationale de la nation basque.

Une conclusion très importante du point de vue des Relations Internationales est le fait qu'avec la formation du premier Gouvernement Basque en 1936, s'est produit le principal saut qualitatif de l’action extérieure. On est passé à une action de type institutionnel, encadrée par la sphère gouvemementale. Dans la mesure où il a eu un gouvernement propre, le Pays Basque s'est converti en acteur de la Société Internationale, exécutant, dans le cadre de son gouvemement représentant le peuple basque, une remarquable action internationale. 


\section{Les indicateurs du modèle d'action extérieure et leur application}

Il est nécessaire de faire remarquer que le problème de l'analyse et de l'interprétation des informations réunies (principalement la bibliographie nationaliste et les textes des dirigeants nationalistes), se résout en appliquant une série d'indicateurscadres qui permettent d'identifier et d'expliquer le modèle d'action extérieure du nationalisme. Je distingue trois types : de doctrine (principes inspirateurs); de programme (objectifs fixés) ; et d’instruments ou auxiliaires.

\section{Les indicateurs sont :}

1) Le contexte historique : pour analyser l'action extérieure, il est indispensable de maintenir les références correspondant au triple cadre : basque, espagnol et international.

2) La prise en compte du contexte et des événements internationaux : en vérifiant de quelle manière, avec quelle intensité et régularité le nationalisme basque, à travers ses publications et ses journaux, a réalisé ses actions extérieures.

3) Les principes inspirateurs : généralement toute action extérieure s'inspire de quelques grandes lignes directrices, idéologico-politiques, qui peuvent être par nature plus ou moins générales, mais qui offrent des lignes directrices au moment de décider ou d'agir.

4) Les objectifs de l'action extérieure : c'est un des éléments les plus significatifs, d'examiner si les objectifs sont définis, ce qu'ils sont, quand ils se dessinent, dans quelle mesure ils sont modifiés, etc., ils nous permettent d'avancer avec des garanties de précision à propos d'un schéma de modèle extérieur.

5) Les alliances internationales : en disposer prouve qu'on a atteint un niveau de développement des échanges suffisamment élevé, en effet, cela suppose qu'on dispose d'accords et de niveaux de coordination avec d'autres interlocuteurs. Cet indicateur inclut aussi l'appartenance à des organisations internationales.

6) Les moyens et les instruments : ils apparaissent au moment où l'action extérieure a déjà dépassé la phase initiale, quand se mettent en place les mécanismes nécessaires pour atteindre certains objectifs fixés. Ils dépendent de deux facteurs préalables : du degré de conscience de l'utilité de se doter de moyens efficaces ; et de la capacité d'organisation humaine et économique dont on se dote pour les soutenir.

7) Les délégations à l'extérieur : ce sont des signes révélateurs du développement international, démontrant l'intérêt que l'on trouve dans une présence permanente dans $d$ autres pays et dans le maintien de liens et de relations avec eux.

8) Les activités et formes de présence internationale : sous cette dénomination, on entend les différentes initiatives et activités pratiques (voyages et visites ; communiqués et discours ; échanges amorcés avec d'autres mouvements, Corps Diplomatique, gouvemements, organisations internationales...; propagande extérieure, etc.).

9) Les aires géographiques : c'est un élément qui illustre l'orientation de la projection extérieure, indiquant vers quelles zones se dirigent les activités et expliquant les raisons de donner priorité aux unes sur les autres.

10) Le processus international : tous les indicateurs cités atteignent une plus grande logique si on en fait une lecture d'ensemble. De plus. pour une juste évaluation du comportement du premier Gouvernement Basque, il faut ajouter deux indicateurs à 
ceux préalablement cités : le cadre juridique de l'action extérieure ; et la structure de l'organisation gouvernementale pour les actions extérieures.

L'application de ce schéma et de ces dix indicateurs se réalise à travers une double lecture : 1) La première est de type synchronique, superposant l'ensemble et chacun des indicateurs sur chaque phase chronologique déterminée, cela nous permet de connaître l'état de l'action extérieure à chaque moment (les conclusions de chaque chapitre sont issues de cette operation) ; 2) La seconde est de type diachronique, juxtaposant chaque indicateur à l'ensemble de toutes les phases chronologiques, ceci nous permet d'apprécier l'évolution de chaque indicateur dans le temps (les déductions de cette seconde lecture constituent les conclusions finales de la thèse).

\section{Présentation d'une action extérieure à travers plusieurs phases chronologiques}

Durant les cinquante années étudiées, on peut constater de manière générale quatre grandes périodes:

1) Un commencement lent de l'action extérieure nationaliste : sans oublier les quelques éléments antérieurs (chapitre 2 «Brèves références aux antécédents de l'action extérieure »), on peut affirmer qu'entre la fin du XIX siècle et le début du XX période qui coincide avec la naissance et la première expression du nationalisme basque, a commencé à se dessiner l'action extérieure, bien que d'une manière peu perceptible et lente (chapitre 3 "Le début des activités extérieures du nationalisme basque, dans la phase araniste (1890-1903) ").

Quelques-uns des indicateurs de l'action extérieure commencèrent peu à peu à prendre forme, comme le fait de prêter attention aux événements internationaux ; comme des petites activités (échanges de télégrammes, quelques voyages); ou encore comme la promotion de l'affiliation extraterritoriale et la désignation de délégués du PNB en Amérique (chapitre 4 « Action extérieure dans la phase post-araniste I. (19031913) : années de faible action extérieure $"$ ).

La volonté d'étendre les relations internationales était encore mise au second plan, les nécessités primordiales des nationalistes étaient autres, dans les domaines de politique et d'organisation. L'euskalerriaco (nationaliste modéré) Eduardo de Landeta le soulignait déjà dans le séminaire Euskalduna en 1909 : " personne ne nous remplace dans la tâche de tisser des relations avec le monde extérieur ». C'est, en conséquence, une phase prématurée pour parler d'un modèle d'action extérieure.

2) Un développement notable de l'action extérieure : aux environs de 1915 jusqu'au début des années 20 ; l'action extérieure connut un grand développement du fait des événements relatifs à la première guerre mondiale et des années de l'après-guerre. Les dirigeants nationalistes prirent pleine conscience de la nécessité de s'ouvrir à l'extérieur, ce qui a entraîné un effort théorique en ce sens, ainsi qu une série d'intitatives.

Une partie des indicateurs du schéma restent valides, en même temps que se rajoutent d'autres. Le modèle, bien qu'incomplet et limité, est en grande partie mis en place (chapitre 5 «Action extérieure dans la phase post-araniste II. (1913-1923) : les premières tentatives d internationalisation dans le cas basque ").

Le plus important est qu'on assiste aux premières tentatives pour obtenir une reconnaissance internationale. On peut citer quelques exemples: la présence du PNB

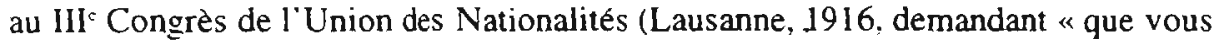
nous reconnaissiez comme parti dans cette grande lutte des petites nations ") : les 
documents, salutations et relations que l'on peut observer après la guerre ; et la revendication d'avoir un espace dans la vie intemationale, jusqu'à obtenir un siège à la Société des Nations. A ce propos José Vilallonga a réfléchi sur la " personnalité internationale » du peuple basque, affirmant que celui-ci « ne peut renoncer à la direction de ses relations extérieures » (Hermes, 1918); alors que Luis de Eleizalde écrivait que «nous devons réussir à ce que la Nation Basque puisse se présenter, dans le respect général, dans l'assemblée des peuples civilisés d'Europe » (Hermes, 1919).

La dynamique extérieure, malgré la division entre Communion Nationaliste Basque (CNB) -secteur nationaliste modéré et en faveur de l'autonomie- et Parti Nationaliste Basque (PNB) -secteur aberkide de ligne politique radicale et indépendantiste-, s'implanta dans le début des années vingt (comme par exemple les contacts avec les républicains irlandais et les solidarités avec les habitants du Rif marocain), mais plus tard il souffrit d'une interruption pendant la dictature de Primo de Rivera. Avec cela, dans la seconde moitié des années vingt, nous percevons quelques signes d'intérêt, comme la montée du pan-européanisme chez les communionistes et les memorandum remis par les « aberkides " à la Société des Nations (chapitre 6 " Action extérieure durant le primorriverisme (1923-1930) : phase de transition ").

3) Réactivation et consolidation de l'action extérieure : la première moitié des années trente, avec la Ile République Espagnole, fut l'apogée pour le thème que nous analysons, réalisant une consolidation presque complète de l'action extérieure nationaliste à tous les niveaux (chapitre 7 « Action extérieure durant la Seconde République (1931-1936) : réactivation des relations internationales »).

Avec les particularités propres à chaque parti (Parti Nationaliste Basque -nationaliste de droite et catholique- et Action Nationaliste Basque -parti nationaliste de gauche, libéral et non confessionnel) et les secteurs (Jagi-Jagi -petit groupe radical-) du nationalisme de l'époque, $s^{\prime}$ affirma la conscience internationaliste, ce qui entraîna des analyses plus nombreuses et plus élaborées; les tâches réalisées vers le monde extérieur occupent une place de première importance ; et se développa un ensemble notable de relations et d'activités intemationales. En 1931, José Antonio Aguirre prévoyait un tel processus : « Aujourd hui, nous allons vers la vie extérieure du pays". Les signes qui l'illustrent sont entre autres les nombreux articles dans la presse nationaliste qui insistent sur la face internationale du problème basque ; la création par le PNB du Secrétariat Général Basque qui assume la propagande à l'extérieur : les voyages dans d'autres pays; l'entrée du PNB au Congrès des Nationalités Européennes avec sa présence dans les réunions annuelles (pour Ramón de Bikuña. cela signifiait donner « un caractère mondial à notre requête "); et l'emploi du slogan «Euzkadi-Europe »(Pays Basque-Europe).

4) Apogée avec une action extérieure basque institutionnelle : presque sans solution de continuité, on entra dans la période 1936-1939, marquée tragiquement par la Guerre Civile espagnole, période pendant laquelle, dans des circonstances minutieusement détaillées dans la thèse, l'action extérieure fait un pas essentiel, dépassant d'une certaine manière le processus initié quelques décennies auparavant (chapitres 8 et 9 « L'action internationale du Gouvernement Basque (1936-1939) : I. L'établissement des relations extérieures institutionnalisées » et «II. Les instruments de base de l'action extérieure. Le rôle des Délégations »).

Avec l'approbation du Statut d'Autonomie du Pays Basque et la constitution du Gouvernement Basque, dans une conjoncture basque, espagnole et internationale très 
complexe, se dessine une nouvelle action extérieure, qui par sa nature, peut être qualifiée de basque et d'institutionnelle, allant plus loin que des relations internationales purement nationalistes et de partis politiques. Pour le nationalisme basque, cela vient couronner une de ses aspirations centrales et pour le reste des formations basques républicaines cela vient contribuer, au-delà des différences, à un moment historique de la projection internationale du Pays Basque. Ce qui fut alors réalisé servit de base à la politique que le cabinet basque effectua dans les années quarante et cinquante.

\section{Le passage qualitatif de l'action extérieure du nationalisme basque, en tant que mouvement politique, au processus international du premier Gouvernement Basque, en tant que gouvernement.}

De fait, dans la période 1936-1939, l'action extérieure effectua une avancée sans précédent avec l'apparition d'une forme d'essor international distincte qualitativement, qui fut menée à bien par le premier Gouvernement Basque, présidé par José Antonio Aguirre, appuyé par le PNB et les formations du Front Populaire (PSOE, ANV, PCE, IR et UR).

Ce changement est particulièrement intéressant pour deux raisons : premièrement, entre les différentes activités nous pouvons voir une certaine continuité, les éléments constitutifs d'une action extérieure nationaliste restent les axes de l'action gouvernementale basque ; et deuxièmement, sans être contradictoire avec ce qui vient d'être dit, ce passage entraîne un changement qualitatif et quantitatif positif pour l'essor international basque.

Avant, jusqu'en 1936, à propos de l'action extérieure spécifiquement nationaliste, nous étions devant le processus d'un mouvement politique, avec une doctrine très concrète et qui réalisait une action extérieure en accord avec des principes inspirateurs, des objectifs, des alliances..., c'est-à-dire, avec des critères propres à tout mouvement politique.

Après, entre 1936 et 1939, il s`agit d'un gouvernement légal, qui par définition possède un caractère institutionnel et qui, en raison des événements historiques connus, eut très vite la chance d'être un « Etat en fonction ", exerçant en conséquence une souveraineté paragée. Le Gouvernement Basque exerça inévitablement dans la pratique, vu la situation exceptionnelle, des fonctions souveraines réservées à l'Etat.

De plus, ce fut un cabinet fortement appuyé politiquement et socialement, avec une représentation du peuple basque dans cette phase historique. ce qui entraîna des conséquences décisives dans le développement de la sphère extérieure, étant donné que la revendication non seulement de légalité mais aussi de légitimité démocratique, contribuait à donner un contenu à un des axes de l'action gouvernementale basque dans le domaine de la politique extérieure.

Il se détache de tout cela une conclusion décisive : le niveau d'action internationale, les tâches entreprises, la structure d'organisation dont elle s'est dotée, les contacts et les relations établies, les délégations implantées avec une reconnaissance politique ou officieuse, la propagande déployée..., eurent une plus grande importance et une plus grande portée pratique que ce qu'avait réussi à obtenir jusque là le nationalisme basque comme mouvement politique.

Euskadi (Pays Basque), comme nation. avec le premier Gouvernement Basque de 1'histoire, entrait de plein droit dans la vie internationale. Ce chemin se traça peu à peu dans les années suivantes. alors que le Gouvernement Basque en exil ne s'est pas dis- 
sous, ni désorganisé, Euskadi continua d'être présente sur la scène internationale, comme une nation sans Etat mais avec un Gouvernement, ce qui lui permit d'accéder à un certain niveau de représentation extérieure. Ceci continua tant que les circonstances, les conditionnements et les valeurs de la Société Internationale le permirent.

\title{
Alexander UGALDE ZUBIRI UPV.EHU
}

\begin{abstract}
NOTES
* Ce travail est un résumé des conclusions de la Thdsc Ductorale élaborée par Alcxander Ugalde Zubiri. intituléc « L'action extcrieure du nationalisme basque (1890-1939) : histoirc. pensée et relations internationales ». soutenue en janvier 1995 à l'Université du Pays Basque, Département des Etudes Intemationales et Sciencc Politique, dirigée par le Dr. Francisco Aldecoa Luzarraga. professeur de Relations Intcrnationales. Publice : La acción exterior del nacionalismo vasco (1890-1939) : historia pensamiento y relaciones internacionales. Oñati. Instituto Vasco dc Administración Pública, 1996. L'auteur remercic Marie Dizicrc. titulairc d'une maîtrisc de Sciences Politiques el Droit Comparé à l'Universite Jean Moulin de Lyon. pour la traduction.
\end{abstract}

